\title{
The Rich Inner Life of the Hydrogen Chain
}

\author{
A one-dimensional chain of hydrogen atoms displays a wide variety of \\ many-body effects-suggesting that the chain can be a useful model \\ system for condensed-matter physics.
}

\section{By Dieter Vollhardt}

$\mathrm{n}$ nature, remarkable effects emerge in systems composed of many interacting particles. The interactions cause correlations between the particles in space and time that cannot be described within a single-particle picture. In solids, in particular, electronic correlations lead to phenomena like high-temperature superconductivity [1] and the emergence of metal-insulator transitions (“Mott transition") [2], for which we don't yet have a comprehensive explanation. Researchers are trying to make progress by studying highly simplified systems that serve as models for the observation of strong correlation effects. One such model is a linear chain of $N$ hydrogen $(H)$ atoms, which captures essential features of the many-electron problem in real materials. By combining several of today's most advanced and accurate computational methods, the Simons

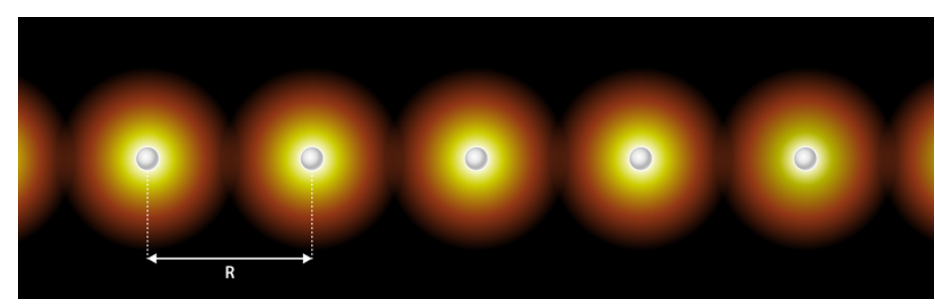

Figure 1: A linear chain of hydrogen atoms, whose nuclei (protons) lie at equal distance R (the colors' brightness maps the electronic density). For large $\mathrm{R}$, as indicated in the figure, the Simons Collaboration found that the system is essentially a collection of isolated hydrogen atoms-each with a single electron-and is insulating. As $\mathrm{R}$ is decreased, the electronic wave functions begin to overlap, and an insulator-to-metal transition occurs at a critical bond length [3].

Credit: APS/Alan Stonebraker
Collaboration on the Many-Electron Problem has now been able to determine, for the first time, fundamental ground-state properties of the $\mathrm{H}$ chain, including spatial correlations between electronic spins in the chain and between the local density of electrons [3]. The results show that this one-dimensional (1D) system exhibits a rich many-body phenomenology, including a new type of insulator-to-metal transition that is fundamentally different from the paradigmatic Mott transition occurring in 3D materials.

Quantum many-particle systems are notoriously difficult to study. This is especially true for electrons in solids. Their investigation requires the solution of the many-electron Schrödinger equation, whose complexity scales exponentially with the number of electrons. The prototypical and simplest microscopic model of electrons in a solid is the Hubbard model, where electrons occupy only one orbital per site and their Coulomb repulsion is assumed to be purely local. Despite its simplicity, the Hubbard model can reproduce typical forms of correlated-electron behavior including magnetism, insulator-metal transitions, and superconductivity.

Even this highly simplified model, however, cannot be solved exactly, except in limited cases (in 1D [4] and in infinite dimensions $[5,6])$. In most cases, "solving" the many-electron problem means obtaining approximate results for the system properties. There are numerous numerical techniques available today, with different ranges of applicability. But they all come with high computational costs and with hard-to-quantify errors.

A more realistic description of materials with correlated electrons must go beyond the Hubbard model, as materials 
involve not only electrons but also ions. The simplest possible system for such an extension is a linear chain of equally spaced $\mathrm{H}$ atoms with one electron per atom (Fig. 1 ). In contrast to the 1D Hubbard model, electrons in the chain can occupy several (in principle, infinitely many) orbitals per site and interact via the long-range Coulomb repulsion. In a previous landmark study, the Simons Collaboration was able to compute a crucial thermodynamic property -the ground-state energy-of an infinite chain of hydrogen atoms [7]. This important advance was obtained with a variety of methods and with extensive cross checks and validations.

These earlier studies provide the methodological foundation for the collaboration's new calculations of more detailed ground-state properties of the $\mathrm{H}$ chain. Deploying an arsenal of numerical and theoretical many-body methods, including Monte Carlo and density-matrix renormalization group techniques, the researchers computed physical properties of the chain, such as the electron localization length (the spatial extension of an electronic wave packet) and the magnetic structure. The researchers investigated how these properties changed as they varied the number of atoms $N$ and the distance between protons $R$. When the results are extrapolated to infinite $N$, the ground state of the system is characterized by only a single parameter $R$.

The researchers found that, for large $R$, the system is a collection of essentially isolated $\mathrm{H}$ atoms, each with a single electron in the atomic $1 s$ orbital. Since each electron is localized around its atom, the system is insulating. This behavior is similar to that predicted by the Hubbard model for the case of strong repulsion, in which case the system corresponds to a spin-1/2 "Heisenberg" chain. Indeed, as the Heisenberg chain, the $\mathrm{H}$ chain at large $R$ has antiferromagnetic correlations that decay over distance with a characteristic power law. In addition, the team observed that the chain undergoes dimerization-the electron distributions around two neighboring ions slightly distort and pair up. This dimerization is reduced as the number of atoms increases. These features are signatures of genuine many-body effects that cannot be explained within one-particle approximations.

As the distance $R$ decreases, the "inner life" of the hydrogen atom-the complexity of its orbitals-starts playing a key role, leading to surprising behavior. Below a critical value of $R$ (about
$85 \%$ of the diameter of a hydrogen atom in its ground state), the chain turns metallic through a fundamentally new type of insulator-to-metal transition. In a 3D material, the well-known Mott transition occurs when electron-hole pairs suddenly unbind, leading to a dramatic screening of the long-range Coulomb interaction. The resulting phase transition is discontinuous, or "first order." A different mechanism is at play for the $\mathrm{H}$ chain. As $R$ decreases, the electronic wave functions start to overlap such that the occupied $1 s$ states mix with the excited states ( $2 s, 2 p$, etc.) to form several energy bands that cross at the Fermi level. This crossing leads to mobile charge carriers, making the system metallic. The numerical simulations indicate that this transition, which is absent in the 1D Hubbard model [4], is continuous, or "second order." It is quite possible that this behavior takes place even in higher dimensions, which would signal a fundamentally new type of Mott transition.

The researchers suggest that their findings might be tested experimentally with ultracold atoms in optical lattices [8], arrays of Rydberg atoms [9], or confined linear carbon chains [10]. Such experimental realizations would provide important contributions to the ongoing study of metallicity in hydrogen at extreme density, which is relevant for understanding astrophysical bodies.

The investigation proves that the $\mathrm{H}$ chain is a fascinating many-body model system, which is now within our theoretical reach, thanks to the advances brought about by collaborative efforts that combine independent methods. Clearly, there is still a long way to go before the approach can be applied to realistic bulk materials. First, computational studies of the $\mathrm{H}$ chain itself should be extended beyond the ground state, exploring excited states, finite temperature, and even nonequilibrium conditions. Most importantly, the step beyond 1D needs to be taken. Here, the experience with systematic investigations of the 2D Hubbard model [11] should be very helpful.

Dieter Vollhardt: Center for Electronic Correlations and Magnetism, University of Augsburg, Augsburg, Germany

\section{REFERENCES}

1. P. A. Lee et al., "Doping a Mott insulator: Physics of high-temperature superconductivity," Rev. Mod. Phys. 78, 17 (2006).

2. N. F. Mott, "Metal-insulator transition," Rev. Mod. Phys. 40, 677 
(1968).

3. M. Motta et al. (Simons Collaboration on the Many-Electron Problem), "Ground-state properties of the hydrogen chain: Dimerization, insulator-to-metal transition, and magnetic phases," Phys. Rev. X 10, 031058 (2020).

4. E. H. Lieb and F. Y. Wu, "Absence of Mott transition in an exact solution of the short-range, one-band model in one dimension," Phys. Rev. Lett. 20, 1445 (1968).

5. W. Metzner and D. Vollhardt, "Correlated lattice fermions in $d=\infty$ dimensions," Phys. Rev. Lett. 62, 324 (1989).

6. A. Georges et al., "Dynamical mean-field theory of strongly correlated fermion systems and the limit of infinite dimensions," Rev. Mod. Phys. 68, 13 (1996).

7. M. Motta et al. (Simons Collaboration on the Many-Electron
Problem), "Towards the solution of the many-electron problem in real materials: Equation of state of the hydrogen chain with state-of-the-art many-body methods," Phys. Rev. X 7, 031059 (2017).

8. I. Bloch et al., "Many-body physics with ultracold gases," Rev. Mod. Phys. 80, 885 (2008).

9. M. Endres et al., "Atom-by-atom assembly of defect-free one-dimensional cold atom arrays," Science 354, 1024 (2016).

10. L. Shi et al., "Confined linear carbon chains as a route to bulk carbyne," Nat. Mater. 15, 634 (2016).

11. J. P. F. LeBlanc et al. (Simons Collaboration on the Many-Electron Problem), "Solutions of the two-dimensional Hubbard model: Benchmarks and results from a wide range of numerical algorithms," Phys. Rev. X 5, 041041 (2015). 